\title{
Qualidade de vida e nível de atividade física de idosos normotensos e hipertensos cadastrados na estratégia de saúde da família
}

\author{
Quality of life and physical activity level of elderly normotensive and \\ hypertensive registered in the family health strategy
}

Paula dos Santos Silva', Ramires Alsamir Tibana² Nuno Manoel Frade de Sousa3, Viviane Daiane Coelho Pinheiro4 , Renata Alves dos Santos ${ }^{4}$, Érick Silva Magalhães Bastos4, Eliane Trevisan, Guilherme Borges Pereira², Jonato Prestes 1,2

\begin{abstract}
Resumo
O objetivo desse estudo foi comparar a qualidade de vida $(\mathrm{QV})$ e o nível de atividade física em idosos normotensos e hipertensos. Trata-se de um estudo quantitativo do tipo transversal realizado em 80 idosos, divididos em dois grupos: hipertensos ( $\mathrm{n}=40 ; 69,5 \pm 13,3$ anos) cadastrados no programa HIPERDIA e normotensos ( $n=40 ; 67,4 \pm 13,5$ anos) cadastrados na estratégia de saúde da família por meio cartazes e folders. A pesquisa foi realizada no domicílio de cada paciente. Para avaliar a QV foi utilizado o questionário World Health Organization Quality of Life e para avaliar nível de atividade física o Questionário Internacional de Atividade Física (IPAQ). O escore total de QV dos normotensos foi melhor em comparação aos hipertensos $(p=0,022)$. O grupo normotenso também apresentou escore no domínio físico superior ao grupo hipertenso ( $p=0,039)$. Para os domínios psicológico, social e ambiental não foram observadas diferenças significativas nos escores $(p>0,05)$. Os dois grupos foram classificados como "sedentários" pelo IPAQ, independentemente dos valores de pressão arterial. Em conclusão, idosos hipertensos possuem pior percepção de QV do que idosos normotensos, enquanto que, todos os idosos foram classificados como sedentários.
\end{abstract}

\section{Palavras-chave}

Idoso; Qualidade de vida; Hipertensão; Aptidão física; Sedentarismo.

\begin{abstract}
The aim of the present study was to compare the quality of life ( $Q L)$ and physical activity level between normotensive and bypertensive elderly subjects. This study was quantitative and cross sectional consisting of 80 elderly men and women, divided into two groups: bypertensive ( $n=40 ; 69.5 \pm 13.3$ years) registered in the HIPERDIA program and normotensive ( $n=40 ; 67.4 \pm 13.5$ years) registered in the strategy of health family by folders. The research was conducted in the home of each patient. The World Health Organization Quality of Life was used to evaluate the $Q L$ and the International Physical Activity Questionnaire (IPAQ) was used to evaluate the levels of physical activity. The total score of $Q L$ among the normotensive group was higher as compared with the bypertensive group $(p=0.022)$. The normotensive group also presented a better score in the physical domain as compared with the hypertensive group ( $p=0.039)$. There were no statistically significant differences in the scores of psychological, social and environmental domains ( $p>0.05)$. All elderly subjects were classified as sedentary according to the IPAQ regardless of the blood pressure values. In conclusion, elderly bypertensive subjects present a poorer perception of QL, while all elderly subjects were classified as sedentary.
\end{abstract}

\section{Keywords}

Elderly; Quality of Life; Hypertension; Physical Fitness; Sedentarism.

\section{Introdução}

A melhora no controle sanitário, evolução da ciência e aumento da expectativa de vida levaram a uma

1 Universidade Católica de Brasília, Pós-Graduação Stricto Sensu em Gerontologia Barreira, BA. Brasil.

2 Universidade Católica de Brasília, Colegiado de Educação Física, Brasília, DF. Brasil. 3 Faculdade Estácio de Sá. Departamento de Educação Física. Laboratório de Fisiologia do Exercício e Medidas e Avaliação, Vitória, ES. Brasil.

4 Faculdade São Francisco de Barreiras, Colegiado de Enfermagem, Barreira, BA. Brasil. inversão do perfil epidemiológico, com consequente redução das doenças infecciosas e aumento significativo das doenças crônicas não-transmissíveis, incluindo a Hipertensão Arterial Sistêmica (HAS) ${ }^{1}$.

Em idosos, a HAS é uma doença altamente prevalente no Brasil, acometendo pelo menos $60 \%$ da população, sendo um fator determinante na morbida$\mathrm{de}^{2}$. A HAS é o principal fator de risco cardiovascular modificável, estando associada a doença arterial co- 
ronariana, doença cerebrovascular, insuficiência cardíaca, doença renal terminal, doença vascular periférica, hipertrofia ventricular esquerda e disfunção diastólica que podem piorar a Qualidade de Vida (QV) de idosos ${ }^{2}$.

Com o aumento da sobrevida dos pacientes com doenças crônicas não-transmissíveis, a QV passou a ser mais valorizada e a importância de sua avaliação foi reconhecida e incorporada nos estudos clínicos ${ }^{3}$. A QV inclui um amplo espectro de domínios, que vão desde a satisfação com a vida, a modelos baseados em conceitos de independência, controle, competências sociais e cognitivas ${ }^{4}$.

Para o idoso hipertenso não-controlado, mais anos vividos podem significar anos de sofrimento, uma vez que se torna necessária a aceitação da doença e a mudança brusca no estilo de vida. Este período pode ser marcado por doenças, com sequelas, declínio funcional e dependência, perda da autonomia, isolamento social e depressão. Por outro lado, se o idoso consegue manter a autonomia e a independência, com boa saúde física, permanecendo ativo e desfrutando de senso de significado pessoal e social, a QV pode ser altamente positiva ${ }^{5}$. A capacidade funcional adquirida por meio do exercício físico é fator chave para independência e melhora da QV dos idosos ${ }^{6}$.

Dessa forma, o presente estudo teve como objetivo comparar a QV e o nível de atividade física em idosos normotensos e hipertensos assistidos na Estratégia de Saúde da Família.

\section{Métodos}

\section{Delineamento do estudo e local de pesquisa}

Trata-se de uma pesquisa quantitativa do tipo transversal. O estudo foi realizado no período de agosto a setembro de 2015 com idosos cadastrados na Estratégia de Saúde da Família nos diferentes bairros da Cidade de Barreiras, Bahia. Colaboraram para o estudo, agentes comunitários de saúde, indicando os domicílios dos idosos bem como seus prontuários com os devidos diagnósticos, quatro graduandos do curso de enfermagem que ajudaram no processo da coleta de dados.

\section{Aspectos éticos}

Os procedimentos do estudo foram aprovados pelo Comitê de Ética em Pesquisa em Seres Humanos da Universidade Católica de Brasília (Resolução 196/96 do Conselho Nacional da Saúde: ${ }^{\circ}$ CAAE 45633515.0.0000.0029) e todos os sujeitos assinaram o Termo de Consentimento Livre e Esclarecido sobre os procedimentos, riscos e benefícios.

\section{População e amostra}

A amostra foi determinada por conveniência e composta por 80 idosos voluntários convidados por meio de folders de divulgação, divididos em dois grupos de 40 participantes em cada, sendo 55 mulheres e 25 homens. Um grupo foi constituído por idosos com HAS, cadastrados necessariamente no Programa "Hiperdia". O Hiperdia destina-se ao cadastramento e acompanhamento de portadores de hipertensão arterial e/ou diabetes mellitus atendidos na rede ambulatorial do Sistema Único de Saúde (SUS), permitindo gerar informação para aquisição, dispensação e distribuição de medicamentos de forma regular e sistemática a todos os pacientes cadastrados. Este programa orienta os gestores públicos na adoção de estratégias de intervenção e permite conhecer o perfil epidemiológico da hipertensão arterial e do diabetes mellitus na população. 
O outro grupo foi constituído por idosos normotensos cadastrados na Estratégia de Saúde da Família. A Estratégia Saúde da Família visa à reorganização da atenção básica no Brasil, de acordo com os preceitos do SUS, e é tida pelo Ministério da Saúde e gestores estaduais e municipais como estratégia de expansão, qualificação e consolidação da atenção básica por favorecer uma reorientação do processo de trabalho com maior potencial de aprofundar os princípios, diretrizes e fundamentos da atenção básica, de ampliar a resolutividade e impacto na situação de saúde das pessoas e coletividades, além de propiciar uma importante relação custo-efetividade.

\section{Critérios de inclusão e exclusão}

Os critérios de inclusão para o estudo foram: idade superior a 60 anos; para o grupo hipertenso estar cadastrado no Hiperdia; para o grupo normotenso estar cadastrado no programa Estratégia Saúde da Família; não apresentar doenças neurológicas e degenerativas, diabetes mellitus, acidente vascular encefálico ou qualquer outra condição que pudesse comprometer a compreensão e capacidade de resposta aos questionários. A desistência ou impossibilidade de qualquer natureza em realizar qualquer uma das etapas do protocolo de pesquisa foram consideradas como critérios de exclusão.

\section{Instrumentos e Procedimentos de Coleta dos Dados}

Após a permissão da enfermeira responsável na Unidade básica de Saúde, aos prontuários dos idosos normotensos e hipertensos foram acessados. Com o suporte dos agentes comunitários de saúde os domicílios dos participantes foram localizados e visitados em horário comercial (8h00-17h00), nos quais os questionários foram aplicados em local reservado.

O questionário World Health Organization Quality of Life (WHOQOL-Bref) validado no Brasil permitiu avaliar a $\mathrm{QV}^{7}$. O WHOQOL-BREF é um instrumento genérico composto por quatro domínios: físico; psicológico; relações sociais; meio ambiente ${ }^{7}$. Os escores variam de 4 a 20 pontos, sendo que o maior valor corresponde à maior $\mathrm{QV}$.

O questionário Internacional de Atividade Física (IPAQ) foi aplicado e classificou o nível de atividade física dos participantes ${ }^{8}$. As perguntas do IPAQ estão relacionadas às atividades realizadas na última semana anterior à aplicação do questionário. Os participantes tiveram seus dados tabulados, avaliados e foram posteriormente classificados nas categorias: Sedentário - Não realiza nenhuma atividade física por pelo menos 10 min contínuos durante a semana; Insuficientemente Ativo - Consiste em classificar os indivíduos que praticam atividades físicas por pelo menos 10 min contínuos por semana, porém de maneira insuficiente para ser classificado como ativos. Para classificar os indivíduos como ativos, são somadas a duração e a frequência dos diferentes tipos de atividades (caminhadas + moderada + vigorosa). Essa categoria divide-se em dois grupos: Insuficientemente Ativo A - Realiza 10 min contínuos de atividade física, seguindo pelo menos um dos critérios citados: frequência - 5 dias/semana ou duração - $150 \mathrm{~min} /$ semana; Insuficientemente Ativo B - Não atinge nenhum dos critérios da recomendação citada nos indivíduos insuficientemente ativos A; Ativo - Cumpre as seguintes recomendações: a) atividade física vigorosa $-\geq 3$ dias/semana e $\geq 20 \mathrm{~min} / \mathrm{sessão;} \mathrm{b)} \mathrm{moderada} \mathrm{ou} \mathrm{caminhada}-\geq$ 5 dias/semana e $\geq 30 \mathrm{~min} / \mathrm{sessão}$; c) qualquer atividade somada: $\geq 5$ dias/semana e $\geq$ $150 \mathrm{~min} / \mathrm{semana}$; Muito Ativo - Cumpre as seguintes recomendações: a) vigorosa $\geq 5$ dias/semana e $\geq 30 \mathrm{~min} /$ sessão; b) vigorosa $-\geq 3$ dias/ semana e $\geq 20 \mathrm{~min} / \mathrm{sessão}$ + moderada e ou caminhada $\geq 5$ dias/ semana e $\geq 30 \mathrm{~min} / \mathrm{sessão.}$ 
A Pressão Arterial (PA) de repouso dos pacientes foi medida seguindo os critérios recomendados pela Sociedade Brasileira de Hipertensão Arterial em todos os pacientes do estudo ${ }^{2}$. Na medida da PA foi utilizado o método auscultatório, com esfigmomanômetro com marcador aneróide (ponteiro) devidamente calibrado e estetoscópio da marca Littmann. A medida ocorre através da oclusão arterial braquial pela inflação do manguito, correlacionando a ausculta dos ruídos de Korotkoff com o valor registrado pelo ponteiro durante a desinflação. Foram realizadas 3 medidas intercaladas com 1 minuto de repouso e utilizado a média de cada participante para as análises posteriores.

As medidas de massa corporal e estatura foram tomadas segundo as técnicas conforme as recomendações do fabricante. A massa corporal foi medida em uma balança da marca Filizola ${ }^{\circledR}$, com capacidade de $150 \mathrm{~kg}$, e a estatura medida em estadiômetro acoplado à balança com capacidade para 1,90 m. O índice de massa corporal (IMC) foi calculado a partir das medidas de peso e de altura utilizando-se a fórmula: $\mathrm{IMC}=$ Massa Corporal $(\mathrm{kg}) /(\text { Estatura })^{2}(\mathrm{~m})$.

\section{Análise estatística}

Os resultados são expressos em média \pm desvio padrão (DP). O teste Shapiro-Wilk foi utilizado para observar a normalidade dos dados. Os dados do questionário IPAQ não apresentaram normalidade e por isso foram avaliados por meio de testes não-paramétricos. Foi utilizado o coeficiente alpha de Cronbach para determinar a confiabilidade do questionário WHOQOL-bref aplicado. Os escores para cada aspecto foram pontuados, segundo sintaxe específica do questionário, em uma escala de 4 a 20 pontos. O valor de 4 pontos significa nenhuma importância e 20 pontos significa total importância. O teste t não pareado foi usado para comparar os escores de QV entre os grupos normotenso e hipertenso. O teste chi-quadrado foi utilizado para observar a possível relação entre a quantidade de idosos praticantes de atividade física e a classificação referente à situação normotensão e hipertensão. O teste $t$ não pareado para dados não-paramétricos foi utilizado para comparar o volume semanal de cada atividade entre os grupos normotenso e hipertenso. O software utilizado foi o SPSS versão 20.0 (Somers, NY, USA) com nível de significância de $\mathrm{p} \leq 0,05$.

\section{Resultados}

A tabela 1 apresenta as características antropométricas e hemodinâmicas dos grupos hipertenso e normotenso. $\mathrm{O}$ grupo hipertenso apresenta maiores valores de IMC ( $\mathrm{p}=0,037)$, PA sistólica $(\mathrm{p}<0,001)$ e PA diastólica $(\mathrm{p}<0,001)$.

A consistência interna do WHOQOL-bref, avaliada pelo coeficiente de Cronbach, é apresentada na tabela 2. Os valores do coeficiente de Cronbach atestam uma consistência interna boa para os 4 domínios em conjunto e para as 26 questões e aceitável ou muito próximo do aceitável para cada domínio analisados de forma isolada.

O escore total de QV dos normotensos foi estatisticamente superior ( $\mathrm{p}=0,022)$ em relação aos hipertensos (tabela 3). Além disso, o grupo normotenso também apresentou escore no domínio físico estatisticamente superior $(\mathrm{p}=0,039)$ ao grupo hipertenso. Para os domínios psicológico, social e ambiental não foram observadas diferenças estatisticamente significativas $(p>0,05)$ nos escores (tabela 3 ).

Considerando o questionário IPAQ, a quantidade de idosos que realizavam caminhada, atividades moderadas ou vigorosas por pelo menos 10 minutos não foi significativamente diferente $(\mathrm{p}>0,05)$ entre os grupos normotenso e hipertenso (tabela 4). A figura 1 apresenta o volume semanal de caminhada, atividades 
TABELA 1 - Características antropométricas e hemodinâmicas dos grupos hipertenso e normotenso (média $\pm D P)$, Barreiras, BA $(n=80)$.

\begin{tabular}{lcc}
\hline Homens & $\begin{array}{c}\text { Normotenso } \\
(\mathrm{n}=40)\end{array}$ & $\begin{array}{c}\text { Hipertenso } \\
(\mathrm{n}=40)\end{array}$ \\
\hline Idade (anos) & $15(37,5)$ & $11(27,5)$ \\
\hline Estatura $(\mathrm{cm})$ & $67,4 \pm 13,5$ & $69,5 \pm 13,3$ \\
\hline Massa corporal $(\mathrm{kg})$ & $161,8 \pm 6,7$ & $160,8 \pm 6,4$ \\
\hline Índice de massa corporal $\left(\mathrm{kg} / \mathrm{m}^{2}\right)$ & $75,6 \pm 13,4$ & $79,7 \pm 16,6$ \\
\hline PA sistólica $(\mathrm{mmHg})$ & $28,7 \pm 3,6$ & $30,6 \pm 5,2^{*}$ \\
\hline PA diastólica $(\mathrm{mmHg})$ & $117,3 \pm 9,9$ & $139,8 \pm 14,6^{*}$ \\
\hline
\end{tabular}

PA, pressão arterial; ${ }^{*} \mathrm{p}<0,05$ diferença significativa entre os dois grupos testada pelo teste t não pareado.

TABELA 2 - Coeficiente de fidedignidade de Cronbach dos domínios e questões, Barreiras, BA ( $n=80$ ).

\begin{tabular}{lcc}
\hline & CC & NI \\
\hline Domínios & 0,814 & 4 \\
\hline Questões & 0,865 & 26 \\
\hline Domínio físico & 0,648 & 7 \\
\hline Domínio psicológico & 0,672 & 6 \\
\hline Domínio social & 0,627 & 3 \\
\hline Domínio ambiental & 0,784 & 8 \\
\hline
\end{tabular}

CC, coeficiente de Cronbach; NI, número de itens.

TABELA 3 - Escores de qualidade de vida geral e dos diferentes domínios dos grupos normotenso e hipertenso (média \pm DP), Barreiras, BA $(n=80)$.

\begin{tabular}{lcc}
\hline & Normotenso & Hipertenso \\
\hline Geral & $15,55 \pm 2,92$ & $14,05 \pm 2,81^{*}$ \\
\hline Domínio físico & $15,14 \pm 2,84$ & $13,63 \pm 3,56^{*}$ \\
\hline Domínio psicológico & $15,02 \pm 2,51$ & $14,17 \pm 3,35$ \\
\hline Domínio social & $14,70 \pm 3,13$ & $14,10 \pm 3,12$ \\
\hline Domínio ambiental & $12,78 \pm 2,75$ & $12,70 \pm 2,63$ \\
\hline
\end{tabular}

${ }^{*} \mathrm{p}<0,05$ diferença significativa entre os dois grupos testada pelo teste t não pareado.

TABELA 4 - Frequência absoluta (n) e frequência relativa (\%) de indivíduos dos grupos normotenso e hipertenso que praticavam caminhada, atividades moderadas ou atividades vigorosas por pelo menos 10 minutos semanais, Barreiras, $B A(n=80)$.

\begin{tabular}{lccc}
\hline & $\begin{array}{c}\text { Normotenso } \\
\mathrm{n}(\%)\end{array}$ & $\begin{array}{c}\text { Hipertenso } \\
\mathrm{n}(\%)\end{array}$ & $\mathrm{X} 2$ \\
\hline Caminhada & $39(97,5)$ & $36(90,0)$ & 0,166 \\
\hline Atividades moderadas & $32(80,0)$ & $27(67,5)$ & 0,204 \\
\hline Atividades vigorosas & $12(30,0)$ & $7(17,5)$ & 0,189 \\
\hline
\end{tabular}

X2, chi-quadrado.

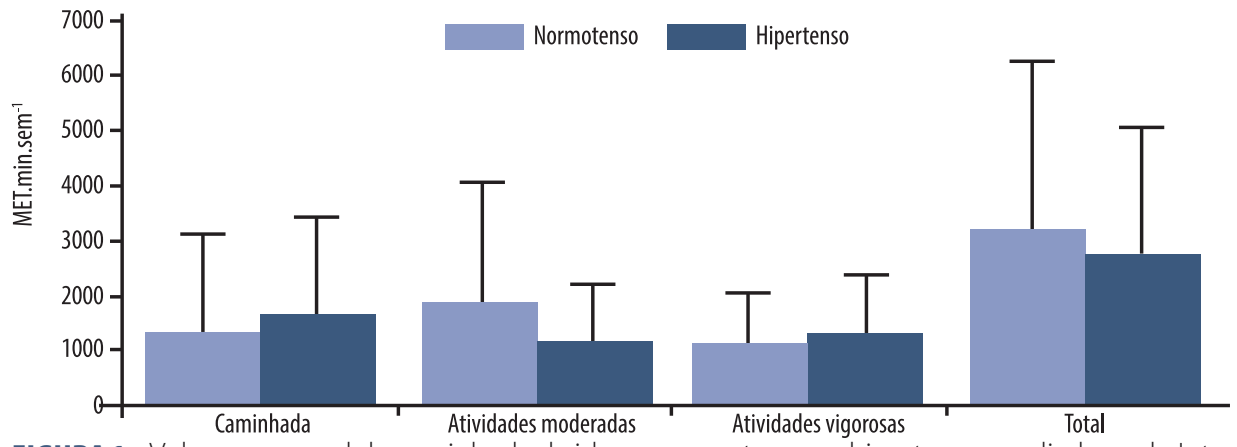

FIGURA 1 - Volume semanal de caminhada de idosos normotensos e hipertensos avaliados pelo International Physical Activity Questionnaire (IPAQ). Teste de Mann-Whitney-Wilcoxon, Barreiras, BA ( $n=80$ ). 
moderadas ou vigorosas por pelo menos 10 minutos e gasto energético total na semana para os grupos normotenso e hipertenso. Não foram observadas diferenças estatisticamente significativas no gasto energético de caminhada $(\mathrm{p}=0,446)$, atividades moderadas $(\mathrm{p}=0,093)$, atividades vigorosas $(\mathrm{p}=0,726)$ ou mesmo no gasto energético total semanal $(\mathrm{p}=0,462)$ entre os grupos estudados. $\mathrm{O}$ mesmo ocorreu para os minutos passados sentado durante a semana (423 $\pm 152 \mathrm{~min}$ para o grupo normotenso e $410 \pm 175 \mathrm{~min}$ para os hipertensos; $\mathrm{p}=0,838)$, entretanto os hipertensos relataram maior tempo sentado nos finais de semana (542 \pm 90 min) em relação ao normotensos (481 $\pm 110 \mathrm{~min}$; $\mathrm{p}=0,008)$.

\section{Discussão}

O objetivo do estudo foi comparar a QV e o nível de atividade física em idosos normotensos e hipertensos assistidos na Estratégia de Saúde da Família. Os resultados demonstraram que os domínios físico e geral da QV do grupo de idosos hipertensos são menores e todos os participantes (grupo hipertenso e normotenso) foram classificados como sedentários.

Ressalta-se a predominância do sexo feminino em ambos os grupos, o que remete que às mulheres teriam uma maior longevidade, demonstrando maior interesse e participação nas atividades sociais, sendo ainda mais cuidadosas com sua saúde e mais vaidosas; e os homens seriam vistos como menos ativos, desleixados e com um envelhecimento mais acelerado?.

O achado sobre o domínio físico nos hipertensos pode estar relacionado há vários fatores que envolvem a doença. Nesse sentido, Tavares et al. ${ }^{12}$ observaram que idosos que possuem várias co-morbidades o domínio físico é o que se apresenta menor escore de QV, relacionando-se a presença de dor e desconforto, alterações do sono, na mobilidade e dependência no uso de medicações e seus efeitos colaterais bem como, realização de tratamento.

As alterações morfofuncionais inerentes ao processo de envelhecimento humano, quando estão associadas a doenças crônicas, podem levar à diminuição da independência física do idoso ${ }^{13}$. Assim, o baixo nível de atividade física possui estreita relação entre doenças crônicas e incapacidade para realização das atividades da vida diária nos idosos, sendo a HAS a condição crônica mais frequente nessa faixa etária. Além disso, Mi et al. ${ }^{14}$ demonstraram que o fato de o paciente saber da doença e do status da sua hipertensão foi negativamente associado com a QV de Chineses que vivem em área rural. Após ajustes para parâmetros socioeconômicos, antropométricos, estilo de vida e co-morbidades, aqueles que sabiam da hipertensão apresentaram piores escores de QV quando comparados ao que não sabiam doença ${ }^{14}$. Outro fator que merece destaque e que pode piorar a QV de idosos hipertensos, é o tratamento contínuo farmacológico para o controle da $\mathrm{PA}^{15}$. Nesse sentido, especula-se que piores escores do domínio físico de QV podem ser associados aos baixos níveis de atividade física observado no presente estudo.

Queiroz et al. ${ }^{16}$ investigaram os efeitos do treinamento de força em idosos e observaram que apesar do processo de envelhecimento reduzir drasticamente a massa, a força e a potência muscular, diminuindo a capacidade de execução das atividades da vida diária, a prática de exercícios de força, pode reverter esse quadro, auxiliando na manutenção da massa muscular e melhorando sua força e resistência, o que possivelmente refletiria sobre o domínio físico da QV.

O mesmo ocorreu no estudo realizado por Benedetti et al. ${ }^{17}$ que pesquisou sobre o nível de atividade física no idoso no Sul do país, onde aplicou-se o mesmo questionário IPAQ para a coleta de dados. O resultado foi que a maior parte dos 
idosos $(93,5 \%)$ eram sedentários em todos os domínios do questionário. O mesmo autor menciona que é frequente a perda de interesse e/ou motivação para aderir a atividades físicas, culturais e sociais, principalmente nos idosos que sofrem de depressão, que acarreta a diminuição nas suas atividades diárias, tornando-os mais sedentários no lar e na sociedade.

Frente a esse contexto, ressalta-se a importância da prática regular de todos os idosos, independente da doença hipertensiva, pois, garante que a prática do exercício físico, além de combater o sedentarismo, contribui significativamente para a manutenção da aptidão física do idoso, seja na sua vertente da saúde como nas práticas funcionais ${ }^{17}$.

Embora esteja clara a importância da prática do exercício físico para a manutenção da saúde, no atual estudo não foi observado correlação do nível de atividade física com a $\mathrm{QV}$, possivelmente porque todos os idosos participantes eram sedentários, independentemente de serem ou não hipertensos.

Quanto às limitações do estudo, alguns pontos merecem destaque. Primeiramente, a amostra do presente estudo não apresenta caráter representativo e recomenda-se que novos estudos com cálculo amostral e poder da amostra fortalecem os achados observados. Apesar das medidas utilizadas neste estudo serem validadas, a utilização de equipamentos mais quantitativos como acelerômetro permitirão uma análise mais detalhada do nível de atividade física. Outro ponto que merece destaque é inserção de novos grupos, como por exemplo, grupo de idosos fisicamente ativos. No entanto, o presente estudo apresenta pontos importantes sobre a qualidade de vida de idosos hipertensos e o sedentarismo dos idosos avaliados por ferramentas simples e válidas nessa população.

Em conclusão, idosos hipertensos possuem pior percepção de QV, especificamente nos domínios geral e físico do que idosos normotensos, enquanto que, todos os idosos foram classificados como sedentários. Novos estudos devem ser realizados investigando a relação existente entre qualidade de vida, nível de atividade física, hipertensão e o impacto de programas de atividade física nestes parâmetros.

\section{Contribuiç̧ão dos autores}

- Paula dos Santos Silva - Desenho experimental, coleta de dados e escrita de todo o artigo;

- Ramires Alsamir Tibana - Desenho experimental e escrita de todo o artigo;

- Nuno Manoel Frade de Sousa - Desenho experimental , análise estatística e escrita dos resultados;

- Viviane Daiane Coelho Pinheiro Coleta de dados, tabulação e escrita da metodologia;

- Renata Alves dos Santos - Coleta de dados, tabulação e escrita da metodologia;

- Érick Silva Magalhães Basto - Coleta de dados, tabulação e escrita da metodologia;

- Eliane Trevisan - Coleta de dados, tabulação e escrita da metodologia ;

- Guilherme Borges Pereira - Desenho experimental e escrita de todo o artigo;

- Jonato Prestes - Desenho experimental e escrita de todo o artigo.

\section{Referências}

1. Casado L, Vianna LM, Thuler LCS. Fatores de Riscos para Doenças Crônicas não transmissíveis no Brasil: uma Revisão Sistemática. Rev Bras Cancerol. 2009;55(4):378-88.

2. Sociedade Brasileira de Cardiologia/ Sociedade Brasileira de Hipertensão/ Sociedade Brasileira de Nefrologia. VI Diretrizes Brasileiras de Hipertensão. Arq Bras Cardiol 2010; 95(1supl.1):1-51. 
3. Carvalho MV de, Siqueira LB, Sousa ALL, Jardim PCBV. A influência da hipertensão arterial na qualidade de vida. Arq. Bras. Cardiol. 2013;100(2): 164-74.

4. Victor C, Scambler S, Bond J, Bowling A. Being alone in later life: loneliness, social isolation and living alone. Rev Clin Geronto. 2000;10:407-17.

5. Nunes MI. Quality of Life in the Elderly Hypertensive. Eur J Prev Cardiol. 2001;8(5):265-9.

6. Gonçalves CG, Guedes MBOG, Lopes JM, Roing JJ, Guedes TSR, Cortez LC de A, et al. Comparação do perfil entre idosos hipertensos e normotensos no município de Belo Horizonte- MG. Cad Educ Saúde Fisioter. 2014;1(1):(1supl.1)80-81.

7. Fleck MPA, Louzada S, Xavier M, Chachamovich E, Vieira G, Santos L, et al. Aplicação da versão em português do instrumento abreviado de avaliação da qualidade de vida "WHOQOL-Bref". Rev Saude Publica. 2000;34(2):178-83.

8. Mazo GZ, Benedetti TRB. Adaptação do questionário internacional de atividade física para idosos. Artigo original. Rev Bras Cineantropom Desempenho Hum. 2010; 12(6):480-4.

9. Areosa SVC, Ohlweiler ZC. O Idoso e os Grupos de Convivência no Município de Santa Cruz do Sul: dados de pesquisa. REDES, Santa Cruz do Sul. 2000;1:179-87.

10. IBGE. Perfil dos Idosos Responsáveis pelos Domicílios. 2000. Disponível em:http://www. ibge.gov.br/home/presidência. Brasília: Comunicação Social; 2002 [citado 2015 Nov 18].

11. Hwang HF, Liang WM, Chiu YN, Lin MR. Suitability of the WHOQOL-Bref for community-dwelling older people in Taiwan. Age Ageing. 2003;32(6):593-600.

12. Tavares DMS, Martins NPF, Dias FA, Diniz MA. Qualidade de vida de idosos com e sem hipertensão arterial. Artigo Original. Rev. Eletr. Enf. (Internet). 2011;13(2):211-8.

13. Santos SSC. Concepções teórico-filosóficas sobre envelhecimento, velhice, idoso e enfermagem geronto-geriátrica. Rev Brasileira de Enfermagem. 2010;63(6):1035-9.

14. Mi B, Dang S, Li Q, Zhao Y, Yang R, Wang D, Yan H. Association Between Awareness of Hypertension and Health-Related Quality of Life in a Cross-Sectional Population-Based Study in Rural Area of Northwest China. Medicine (Baltimore). 2015;94(29):e1206.

15. Trevisol DJ, Moreira LB, Fuchs FD, Fuchs SC. Health-related quality of life is worse in individuals with hypertension under drug treatment: results of population-based study. J Hum Hypertens. 2012;26(6):374-80.

16. Queiroz ACC, Kaneguzuku H, Forjaz LM. Efeitos do treinamento resistido sobre pressão arterial de idosos. Arq. Bras. Cardiol. 2010;95(1):135-40.

17. Benedetti TRB, Borges LJ, Petroski EL, Gonçalves LHT. Atividade física e estado de saúde mental de idosos. Rev Saúde Pública. 2008;42(2):302-7.

ENDEREÇO PARA

CORRESPONDÊNCIA

JONATO PRESTES

jonatop@gmail.com
Programa de Pós Graduação Stricto

Sensu em Educação Física, Universidade

Católica de Brasília. Q.S. 07, Lote 01 -

Bloco G. CEP: 71966-700

Águas Claras, Taguatinga - DF, Brasil

Tel: 0021-55-61 - 3356-9350.
RECEBIDO

12/02/2016

APROVADO 16/02/2016 